\title{
Tercerización del trabajo y sobreexposición a radiación ionizante en postulantes a services de minería
}

\author{
AUGUSTO RAMÍREZ \\ Médico del Trabajo. American College of Occupational and Environmental Medicine.
}

\begin{abstract}
RESUMEN
OBJETIVO: Determinar la cantidad de exámenes de ingreso que se toma en un año a los postulantes a trabajo en minería por la modalidad de services y cuantificar así el número de radiografías de tórax a que se les somete. DISEÑO: Estudio prospectivo descriptivo. MATERIAL Y MÉTODOS: Se estudia a un grupo de postulantes a trabajo por la modalidad de service en minería, verificándose el número de exámenes pre ingreso y otras exposiciones a radiación equis derivadas de radiografías diagnósticas por accidentes o enfermedades y le suma la cantidad de radiación ionizante natural recibida por laborar en tajo abierto y a gran altura. RESULTADOS: El $45 \%$ de los examinados toma un examen de ingreso al año, $29 \%$ dos, $12 \%$ tres, $13 \%$ entre cuatro y seis y a 5 postulantes se les toma examen de ingreso 7 veces en el año. Al calcular el promedio pesado anual de dosis equivalente efectiva acumulada de radiación ionizante, se encuentra que llega a 169 mili Rem/ año y el promedio pesado de riesgo de contraer cáncer se halla en $289 \times 10^{-6}$ exámenes. CONCLUSIÓN: Se recomienda racionalizar el número de exámenes pre empleo en esta modalidad de trabajo por tercerización.
\end{abstract}

Palabras clave: Radiografía; radiación ionizante; trabajo; minería.

\section{OUTSOURCING AND IONIZING RADIATION OVEREXPOSURE IN APPLICANTS TO MINING SERVICES LABOR SUMMARY}

OBJECTIVE: To determine the number of applicant examinations taken in one year and quantify the number of thoracic X-rays subjected. DESIGN: Prospective descriptive study. MATERIAL AND METHODS: In 400 applicants to work by the modality of services in the Peruvian mining the quantity of applicant exams taken in the previous immediate year to the current examination and the number of chest X-rays in a year were determined. It also verifies additional $\mathrm{X}$-ray exposition due to diagnostic exams derived from accidents or diseases and the quantity of natural ionized radiation received from open pit and high altitude work. RESULTS: Forty-five per cent of men examined take one applicant work examination per year, 29\% two, $12 \%$ three, $13 \%$ between four and six and in 5 applicants seven applicant work exams were taken during the year. By calculating the annual weighted equivalent accumulative dose of ionizing radiation an average of $169 \mathrm{mRem} /$ year was found, with a total weighted risk to develop cancer of 289 x $10^{-6}$ examinations. CONCLUSION: To limit the number of applicant work examinations in this outsourcing work modality is recommended.

Key words: Radiography; radiation ionizing; work; mining.

\footnotetext{
Correspondencia:

Dr. Augusto V. Ramírez

Las Mandarinas 210 Ap. 306

Lima 12, Perú

E-mail: augustovram@yahoo.es
} 


\section{INTRODUCCIÓN}

Que el uso indiscriminado de radiación equis (rayos X) es nocivo para el ser humano se sabe casi desde el descubrimiento de Roentgen. Cada día hay nuevas evidencias de que cualquier exposición a radiación ionizante, por mínima que sea, tiene consecuencias adversas para el organismo humano.

La OMS no ha establecido valor límite umbral (TLV, por sus siglas inglesas threshold limit value) y más bien sostiene que "ningún estudio realizado evidencia la existencia de límite de tolerancia para una dosis mínima de radiación. En el caso de exposiciones mínimas a pequeñas dosis ... los efectos carcinogénicos y genéticos parecen ser acumulativos" $\left({ }^{1,2}\right)$.

Los rayos equis producen dos tipos de efectos a nivel celular:

Ectocásticos, aquellos que con la dosis varían en frecuencia pero no en severidad: mutagénesis, carcinogénesis y teratogénesis.

No ectocásticos, efectos que con la dosis varían en frecuencia y en severidad, tales como eritema de la piel, deterioro de la fertilidad, depresión de la hematopoyesis $\left({ }^{3}\right)$.

Los daños se extienden a la descendencia del individuo. Estudios epidemiológicos y de investigación en exposiciones masivas a radiación ionizante- sea por accidente, por experimentación en animales o por estudios longitudinales en habitantes expuestos a la explosión de bombas atómicas- demuestran que, además del daño sobre el individuo, hay daño genético en la descendencia, en humanos después de 4 generaciones y en animales de experimentación aún después de 10. Entre las manifestaciones deletéreas destacan por su significación la esterilización celular, aberraciones cromosómicas, mutaciones genéticas y cáncer $\left({ }^{4}\right)$.

La magnitud, significancia e intervalo entre exposición y aparición del efecto nocivo depende de las características físicas de la fuente radioactiva y del tipo de tejido alcanzado por la radiación. Por otro lado, el beneficio obtenido con el examen radiográfico depende en mucho de la preparación y experiencia del médico que hace el diagnóstico, del técnico que toma la placa y de la circunstancia del examen, es decir, si es utilizado para el diagnóstico, seguimiento o tratamiento de una enfermedad, si es un examen preventivo o si es voluntario u obligatorio $\left.{ }^{5,6}\right)$.

En salud ocupacional, el uso de radiación ionizante se valora desde dos puntos de vista, uno la protección del trabajador que opera el equipo y otro, los efectos que tiene sobre el trabajador examinado. En trabajadores expuestos a bajos niveles de radiación ionizante (radiólogos, técnicos en $\mathrm{Rx}$, pintores de esferas luminosas en relojes, etc.), hay incremento del número de cánceres, pero no se ha determinado si esto pueda atribuirse solamente a una dosis única previa o a la acumulación de pequeñas dosis consecutivas o espaciadas $\left({ }^{7}\right)$.

Kitabatake, Japón 1968, luego de un estudio retrospectivo de 40 millones de foto radiografías de tórax, cuestiona su utilidad para diagnosticar tuberculosis, cáncer u otras enfermedades, pues con este número de exámenes diagnostica $0,096 \%$ de tuberculosis curables, $0,002 \%$ de cánceres curables y $2,5 \%$ de otras enfermedades $\left({ }^{8}\right)$. Sobre este mismo estudio, Gregg indica que, estadísticamente, al tomar 40 millones de radiografías, es posible se hayan producido 46 casos de leucemia, 7 cánceres de pulmón y 150 enfermedades genéticas mortales para las primeras 10 generaciones $\left({ }^{9}\right)$.

En Brasil, Noguiera halla que en 17638 exámenes con foto radiografía de tórax sólo se diagnostica 58 casos de tuberculosis $(0,3 \%)$ y concluye que 17572 personas fueron sometidas a radiación ionizante innecesariamente $\left({ }^{10}\right)$.

Piexoto $\left({ }^{11}\right)$ estudia la cantidad de radiación que indirectamente absorben otros órganos por cada radiografía de pulmones que se toma. A cada órgano le asigna un peso específico, de acuerdo a su mayor o menor sensibilidad a la radiación, tal como lo hacen Hashizume y 
Maruyama $\left({ }^{12}\right)$, quienes calculan la dosis equivalente efectiva $(H t x W t)$.

En el siguiente cuadro apreciamos el resumen de dicho estudio:

\begin{tabular}{lcc}
\hline Órgano & $\begin{array}{c}\text { Dosis recibida: } \\
\mathrm{H} \mathrm{t}^{*}\end{array}$ & $\begin{array}{c}\text { Especificidad de } \\
\text { órgano: } \mathrm{W} \mathrm{t}^{\dagger}\end{array}$ \\
\hline Gónadas & $4(\mathrm{M}: 1-3$, & 0,25 \\
Mamas & $20 \quad \mathrm{~F}: 3-10)^{\ddagger}$ & \\
Médula ósea & 50 & 0,15 \\
Pulmones & 160 & 0,12 \\
Tiroides & 40 & 0,12 \\
Superficie ósea & 100 & 0,03 \\
Piel & 70 & 0,03 \\
\hline
\end{tabular}

* Dosis recibida por cada órgano cuando se toma una radiografía de pulmones $=H t$

$\dagger$ Peso específico por sensibilidad de cada órgano: $W t$

* Rango por sexo, $M=$ Masculino, $F=$ Femenino

Luego calcula el riesgo de contraer cáncer, tomando como base la dosis equivalente efectiva para otros exámenes radiográficos, de los cuales hemos tomado los que interesan a nuestro estudio y cuya síntesis vemos en la siguiente relación.

\begin{tabular}{lcc}
\hline Tipo de examen & DEEA Total & \\
\hline Columna lumbosacra & 170 & 21,0 \\
Abdomen simple & 100 & 13,0 \\
Pulmones & 12 & 1,0 \\
Miembro superior & 8 & 1,0 \\
Miembro inferior & 4 & 0,5 \\
Parrilla costal & 76 & 10,0 \\
Dental & 3 & 0,5 \\
Cráneo & 34 & 4,0 \\
\hline
\end{tabular}

* DEEA = Dosis equivalente efectiva año en miliRem

+ Riesgo total $=$ Factor de riesgo (FR) calculado por un millón de exámenes

Con estos datos analiza hallazgos de Hashizume $\left({ }^{12}\right)$ y Jankosky $\left({ }^{13}\right)$ y relaciona dosis equivalente efectiva anual (DEEA) con ries- go de contraer cáncer (factor de riesgo: FR) expresado en número de casos de cáncer por millón de radiografías tomadas; establece que el examen de tórax para corazón y pulmones tiene una frecuencia anual de 505,6 exámenes por 1000 habitantes y una DEEA de 38 mili Rem (mRem), con lo que determina un FR igual a 4 casos por millón de exámenes. A pesar que este riesgo parece bajo frente a exámenes radiográficos de otros órganos (columna p ej.), la verdad es que por la gran cantidad de radiografías de tórax tomadas el riesgo se incrementa.

La International Commission on Radiological Protection (ICRP) considera que el factor de riesgo cáncer inducido por radiación ionizante es de $1,25 \times 10^{-6}$ casos por persona por unidad equivalente de dosis en mRem, de ahí que por los $12 \mathrm{mRem}$ de cada radiografía, serían esperados 1,6 casos de cáncer inducido en cada millón de personas sometida a un examen de tórax/año. Ergo, si lo multiplicamos por 3, el riesgo aumenta a 4,8 , si por 7 a 11,2 . Con la radiografía estándar de tórax, la dosis recibida es de 0,012 Rem, pero si el examen se hace con foto radiografía, el riesgo aumenta por la mayor cantidad de radiación liberada 0,259 Rem, y es menos efectiva, ya que su menor resolución la hace de poca utilidad en el diagnóstico de neumoconiosis o de tuberculosis $\left({ }^{10,13-16}\right)$.

La American Cancer Society suspende su recomendación de hacer una radiografía anual de tórax en asintomáticos porque "no se observa ninguna mejoría en la mortalidad por cáncer de pulmón derivada de esta práctica". Y un panel del National Center for Devices and Radiological Health de la FDA (EU de A) elabora, con la participación de ACR, ACO\&G, la entonces AOMA y la ATS, 5 criterios para evitar la indicación rutinaria del examen radiográfico de tórax:

1. "Los estudios radiográficos de tórax deben ser suspendidos en mujeres de edad fértil, cuando existan otros procedimientos diagnósticos aplicables... 
2. Los exámenes radiográficos de tórax deben suspenderse en... mujeres con sospecha de embarazo o gestantes.

3. Se suspenden los exámenes radiográficos de tórax en pacientes que reciban terapia con cualquier elemento radiactivo...

4. Los exámenes radiográficos de tórax realizados para la admisión a un trabajo ... han mostrado poco valor clínico, por lo que no se justifica el valor de su uso.

5. Deben ser suspendidos los exámenes radiográficos de tórax en ... los exámenes rutinarios no relacionados a exposiciones ocupacionales que puedan afectar la función pulmonar" $\left({ }^{17}\right)$.

El ICRP dice que "existe relación linear sin límite de tolerancia y la posibilidad de que el efecto sea acumulativo cuando se trata... de las radiaciones" $\left.{ }^{14}\right)$. El Committee on the Biological Effect of Ionizing Radiation (CBEIR) sostiene que "el uso médico de alta dosis instantánea de radiación es la causa de la mayor dosis elevada de irradiación en la totalidad de los órganos de los individuos expuestos, aunque sea en exposiciones muy cortas" $\left({ }^{18}\right)$. Así, el riesgo carcinogénico puede ser estimado tan sólo por interpolación, basada en presunciones acerca de la relación radiación/ dosis $\left({ }^{19}\right)$. La consistencia de la relación dosis/ incidencia de cáncer sugiere que la exposición a dosis sucesivas, pequeñas y espaciadas de radiación ionizante han comprobado ser aditivas en sus efectos carcinogénicos en mama y la incidencia se incrementa sin función umbral (non threshold) de la dosis acumulativa $\left({ }^{20}\right)$.

Si bien la incidencia se incrementa con la dosis, la magnitud del incremento parece ser el mismo si la dosis es dada de una sola vez, como en el accidente de Chernobyl $\left({ }^{21}\right)$, en 4 ó 5 días, como en el tratamiento de mastitis posparto $\left({ }^{22}\right)$, o con intervalo de varios meses, como en pilotos de avión o en pintores de esferas luminosas $\left({ }^{23}\right)$.

En el Perú, la radiografía de pulmones -en placa 14 x 14", llamada estándar, o en foto radiografía-, se usa desde hace más de 50 años en los reconocimientos médicos periódicos de ingreso o de salida del trabajo, principalmente en empresas mineras, donde la prevalencia de neumoconiosis es alta $\left({ }^{24}\right)$. En minería, la ley obliga a los empleadores a tomar un examen de ingreso al trabajo para establecer el estado de salud del nuevo trabajador. Los exámenes preocupacionales y luego los periódicos incluyen radiografía PA de tórax orientada principalmente a buscar neumoconiosis o tuberculosis $\left({ }^{25}\right)$.

Sucede que, con la "modernización" del sistema de labor, aparece la tercerización del trabajo, cuyo fin original es contratar personal eventual o temporal para tareas no ligadas directamente con la actividad principal de la empresa, como p. ej. seguridad de planta, hostería, transporte, etc. $\left({ }^{26,27}\right)$. El inicio de este proceso da lugar a la aparición de services y los que idean el modelo proponen contratar personal temporal en alrededor de $20 \%$ de la planilla de las empresas para cumplir trabajos complementarios, mientras que $80 \%$ seguiría como personal estable en el trabajo principal $\left({ }^{28}\right)$.

Sin embargo, esta noción inicial se desnaturaliza y hoy vemos con preocupación cómo los porcentajes se han invertido, con el agravante que estos trabajadores tercerizados por ser temporales son cancelados, generalmente cada 3 meses. Aunque he visto cancelar "por término de obra" a los 6 u 8 días de iniciar su labor, y como quiera que la empresa siempre va a necesitar hacer su trabajo principal con ellos, se ve en la necesidad de volver a contratarlos a través de "otro" service, que muchas veces es el mismo que tan sólo cambió de razón social. Para lo cual el trabajador es obligado a tomar un nuevo examen pre empleo, que lógicamente conlleva nueva radiografía de pulmones, así hayan transcurrido 15 días, 1 ó 2 meses del último examen radiográfico. Y cuando el trabajador tiene la oportunidad de preguntar por qué otro examen, la respuesta es "para cambio de fotocheck" frente a lo cual al trabajador le quedan 2 caminos: o lo cambia, lo cual implica nuevo examen médico y nueva radiografía de pulmones, o sin el 
fotocheck "nuevo" no ingresa a trabajar a la Empresa.

Justamente, el presente estudio busca establecer la frecuencia de exposición a radiación ionizante tipo rayos $\mathrm{X}$ y otras en postulantes a quienes se les exige un examen preocupacional cada vez que intentan ingresar a un service para laborar y que por la modalidad de este trabajo se repite varias veces en un año. Para ello, nos planteamos un estudio prospectivo descriptivo de 400 postulantes a este tipo de trabajo, averiguamos otras exposiciones a rayos $\mathrm{X}$ en el mismo lapso derivadas de exámenes radiográficos adicionales, y le sumamos la exposición a radiación natural recibida por trabajar en alturas superiores a $4000 \mathrm{msnm}$. Luego calculamos la dosis equivalente efectiva de exposición anual y el factor de riesgo para contraer cáncer.

\section{MATERIAL Y MÉTODOS}

El objetivo del estudio fue investigar el número de radiografías de pulmones tomadas a los postulantes a trabajo en services durante un año cumplido a la fecha en que los examinamos. El dato lo obtuvimos por el número de exámenes de ingreso en este periodo. Con la anamnesis averiguamos otras exposiciones a rayos equis que el postulante hubiera tenido como consecuencia de accidentes o enfermedades. En el análisis también consideramos la exposición a radiación ionizante natural, determinada por la forma de trabajo en tajo abierto (open pit), que es a la intemperie y en alturas superiores a $4000 \mathrm{msnm}$. Con la magnitud de la exposición y con la ecuación de Hashizume y Maruyama $\left({ }^{12}\right)$, calculamos la dosis equivalente efectiva de exposición anual al momento del examen y el factor riesgo de contraer cáncer por estas exposiciones.

\section{Tipo de estudio}

El estudio se planteó como prospectivo y descriptivo. El grupo investigado comprendió a 400 postulantes a trabajo en services, 396 hombres y 4 mujeres, examinados en un centro de exámenes ocupacionales en la ciudad de Cajamarca, Perú, en los meses de marzo, abril y mayo del año 2001 , de acuerdo a la legislación minera vigente al momento del examen $\left({ }^{25}\right)$.

Se incluyó a todos los examinados sin criterios de exclusión y la acumulación de casos se hizo progresivamente hasta completar los 400 . En 193 postulantes examinados se cotejó el dato del número de exámenes anteriores con los services en que habían trabajado.

\section{RESULTADOS}

La Tabla 1 muestra el número de radiografías de pulmones que los postulantes a trabajo tomaron durante el año inmediato anterior al examen. Para $45 \%$ de los examinados fue su primera radiografía, para $29 \%$ la segunda, para $12 \%$ la tercera y, al final de la tabla, vemos que para 5 postulantes fue la sétima en un año. La Tabla también calcula la dosis efectiva equivalente por estas exposiciones y el factor de riesgo individual de contraer cáncer.

Tabla 1.- Número de radiografías por año por dosis equivalente efectiva por riesgo. ( $n=400: 396$ hombres, 4 mujeres)

\begin{tabular}{lrrcc}
\hline $\begin{array}{l}\text { Radiografías de } \\
\text { pulmones/año }\end{array}$ & $\mathrm{n}$ & $\%$ & DEEA $^{*}$ & $\begin{array}{c}\text { Riesgo } \\
\text { individual }^{\dagger}\end{array}$ \\
\hline Una & 181 & 45 & 12 & 1,6 \\
Dos & 115 & 29 & 24 & 3,2 \\
Tres & 48 & 12 & 36 & 4,8 \\
Cuatro & $\mathbf{2 3}$ & $\mathbf{6}$ & $\mathbf{4 8}$ & $\mathbf{6 , 4}$ \\
Cinco & 12 & 3 & 60 & 8,0 \\
Seis & 16 & 4 & 72 & 9,6 \\
Siete & 5 & 1 & 84 & 11,2 \\
Totales y medias & 400 & 100 & 48 & 6,4 \\
\hline
\end{tabular}

* DEEA = Dosis equivalente efectiva año en miliRem $\left({ }^{11}\right)$.

${ }^{\dagger}$ Riesgo individual $=N^{o}$ de casos de cáncer esperado en $10^{6}$ exámenes por radiografía $\left({ }^{11}\right)$.

La Tabla 2 muestra la distribución por ocupación actual de los examinados. Apreciamos 
Tabla 2.- Ocupación actual del postulante.

$$
(\mathrm{n}=400)
$$

\begin{tabular}{lrr}
\hline Ocupación actual & $\mathrm{n}$ & $\%$ \\
\hline Agricultor & 9 & 2,3 \\
Albañil & 67 & 16,8 \\
Armador & 37 & 9,3 \\
Chofer & 34 & 8,5 \\
Contador público & 3 & 0,8 \\
Ingeniero de seguridad & 5 & 1,3 \\
Ingeniero de sistemas & 2 & 0,5 \\
Mecánico & 63 & 15,0 \\
Mecánico soldador & 32 & 8,0 \\
Montajista & 52 & 13,0 \\
Operador de equipo pesado & 29 & 7,0 \\
Profesor educación superior & 1 & 0,3 \\
Profesor educación secundaria & 9 & 2,3 \\
Señalero & 10 & 2,5 \\
Sociólogo & 3 & 0,8 \\
Soldador & 27 & 6,8 \\
Técnico mecánica industrial & 2 & 0,5 \\
Técnico metalurgista & 9 & 2,3 \\
Técnico químico & 2 & 0,5 \\
Técnico seguridad & 5 & 1,3 \\
Total & 400 & 100,0 \\
& &
\end{tabular}

Nota: La ocupación actual del postulante no es necesariamente el cargo al que postula, ya que salvo los ingenieros, los demás fueron para trabajar como "obreros", "peones"o "ayudantes" (sic). que $63 \%$ declaró oficios afines a construcción civil; así 251 dijeron ser albañil, armador, mecánico, soldador o montajista. Mientras que al otro extremo, 1 era profesor de educación superior y en el intervalo encontramos sociólogos, choferes, agricultores, técnicos superiores e ingenieros. Puntualizamos que la ocupación actual del postulante no fue necesariamente el puesto al que aplicaba en el service, ya que, salvo los ingenieros, los demás postularon para "obreros", "peones" o "ayudantes" (sic).

En la Tabla 3, con la ecuación de Hashizume y Maruyama ( ${ }^{12}$ ) calculamos la dosis efectiva equivalente y el factor de riesgo adicional para contraer cáncer por exploraciones radiográficas realizadas por otros motivos.

Por último, la Tabla 4 muestra el consolidado de los 3 tipos de riesgos: número de exámenes radiográficos de pulmones por ingreso al trabajo, número de exámenes radiográficos adicionales más la dosis de radiación natural para trabajo en alturas mayores a $4000 \mathrm{msnm}$ (media de radiación natural recibida: 77,5 mRem/ año), los integra y calcula la dosis equivalente efectiva anual, riesgo adicional, dosis total y riesgo total.

Tabla 3.- Riesgo adicional por otros exámenes radiográficos. ( $\mathrm{n}=95: 95$ hombres, 0 mujeres $)$

\begin{tabular}{|c|c|c|c|c|c|}
\hline Tipo de examen & $\mathrm{n}$ & $\%$ & Dosis por $\mathrm{Rx}$ & DEEA por \# Rx & Riesgo adicional \\
\hline Columna lumbosacra & 10 & 10 & 170 & 1700 & 210,0 \\
\hline Abdomen simple & 2 & 2 & 100 & 200 & 26,0 \\
\hline Pulmones & 16 & 17 & 12 & 192 & 16,0 \\
\hline Miembros superiores & 18 & 19 & 8 & 144 & 18,0 \\
\hline Miembros inferiores & 12 & 13 & 4 & 48 & 6,0 \\
\hline Parrilla costal & 13 & 14 & 76 & 988 & 130,0 \\
\hline Dental & 21 & 22 & 3 & 63 & 10,5 \\
\hline Cráneo & 3 & 3 & 34 & 102 & 12,0 \\
\hline Totales y medias & 95 & 100 & 51 & 430 & 52,0 \\
\hline
\end{tabular}

Dosis por $R x=$ Dosis administrada por tipo de radiografía adicional en mili Rem DEEA por \# Rx = Dosis equivalente efectiva acumulada por número de radiografías adicionales Riesgo adicional $=$ Riesgo adicional de cáncer por $10^{6}$ exámenes $\left({ }^{11}\right)$. 
Tabla 4.- Exposición total en gran altura por número de exámenes año por dosis adicional por riesgo total. $(n=95)$

\begin{tabular}{|c|c|c|c|c|c|c|c|c|c|c|c|c|c|}
\hline $\mathrm{N}^{\circ}$ Rxs de ingreso: & Una & Dos & Tres & Cuatro & Cinco & Seis & Siete & $\begin{array}{l}\text { Total Rx } \\
\text { adicio- } \\
\text { nales }\end{array}$ & DEEA & $\begin{array}{c}\text { Dosis } \\
\text { radiación } \\
\text { natural }\end{array}$ & $\begin{array}{l}\text { Riesgo } \\
\text { adi- } \\
\text { cional }\end{array}$ & $\begin{array}{l}\text { Dosis } \\
\text { promedio } \\
\text { pesada }\end{array}$ & $\begin{array}{r}\text { Riesgo } \\
\text { pesado } \\
\text { Total }\end{array}$ \\
\hline Columna lumbosacra & 0 & 2 & 1 & 2 & 3 & 2 & 1 & 11 & 210 & 77,5 & 37,0 & 287,5 & 407,0 \\
\hline Abdomen simple & 1 & 0 & 0 & 1 & 0 & 0 & 0 & 2 & 26 & 77,5 & 13,4 & 103,5 & 26,8 \\
\hline Pulmones & 1 & 0 & 5 & 2 & 3 & 4 & 0 & 15 & 16 & 77,5 & 12,0 & 93,5 & 180,0 \\
\hline Miembro superior & 0 & 0 & 1 & 3 & 11 & 2 & 1 & 18 & 18 & 77,5 & 12,0 & 95,5 & 216,0 \\
\hline Miembro inferior & 0 & 1 & 0 & 1 & 6 & 2 & 1 & 11 & 48 & 77,5 & 16,0 & 125,5 & 176,0 \\
\hline Parrilla costal & 0 & 1 & 3 & 2 & 6 & 1 & 0 & 13 & 390 & 77,5 & 61,0 & 467,5 & 793,0 \\
\hline Dental & 5 & 7 & 5 & 2 & 2 & 0 & 1 & 22 & 10 & 77,5 & 11,0 & 88,0 & 242,0 \\
\hline Cráneo & 0 & 0 & 1 & 0 & 1 & 1 & 0 & 3 & 12 & 77,5 & 11,0 & 89,5 & 268,5 \\
\hline Totales y medias & 7 & 11 & 16 & 13 & 32 & 12 & 4 & 95 & 91 & 77,5 & 22,0 & 169,0 & 289,0 \\
\hline
\end{tabular}

DEEA = Dosis equivalente efectiva adicional año, debida a radiografías adicionales, expresada en mili Rem (mRem).

Dosis radiación natural = Dosis de radiación natural calculada para altura superior a $4000 \mathrm{msnm}$ y expresada en mRem (32). Dosis promedio pesada = Dosis equivalente efectiva promedio pesada de radiación por radiografía adicional más radiación natural en mRem

Riesgo adicional $=$ Riesgo adicional para contraer cáncer por radiación natural $x 10^{6}$ exámenes.

Riesgo pesado total $=$ Promedio pesado del riesgo total para contraer cáncer $x 10^{6}$ exámenes.

\section{DISCUSIÓN}

Roentgen y la mayoría de sus colaboradores murieron por sarcoma o leucemia causados por exposición en forma empírica a radiación equis ${ }^{(1)}$. Actualmente, el uso de la radiación equis ha sido limitado y racionalizado, principalmente por sus efectos dañinos sobre el individuo y su descendencia $\left({ }^{2-4}\right)$.

En Salud y, particularmente en Salud Ocupacional, el problema tiene varias implicancias para el trabajador, sobre todo cuando busca o cambia de empleo dentro del nuevo sistema laboral por terceros, llamado comúnmente services, en el que tiene que someterse a exámenes pre empleo con sorprendente frecuencia, de manera que no es excepcional encontrar trabajadores a quienes durante el lapso de un año se les somete a más de seis exámenes radiográficos de tórax con placa estándar o con foto radiografía, amen de otras exposiciones por accidentes o enfermedades. Si bien la radiación ionizante es un riesgo para la salud física del trabajador tercerizado, existen además otros riesgos ya descritos, ora para su salud mental $\left({ }^{29}\right)$ ora para su salud social $\left({ }^{30}\right)$, pero que no son objeto de este estudio.

Peixoto cuantifica la cantidad de radiación que absorben otros órganos cada vez que se toma una radiografía de pulmones y a cada uno le asigna un peso específico, de acuerdo a su mayor o menor sensibilidad $\left({ }^{11}\right)$.

Aunque el problema parece simple no lo es, ya que al efecto de la dosis de rayos equis dada en los exámenes pre empleo, se agregan otras recibidas para el diagnóstico de enfermedades o accidentes, pues el trabajo minero es de alto riesgo y los accidentes tienen prevalencia elevada $\left({ }^{24}\right)$, por lo que el diagnóstico traumatológico y de otras enfermedades incrementan la exposición a rayos $\mathrm{X}$.

Aun cuando, en la actualidad, las medidas de protección para el operador de equipos de rayos equis son en la mayoría de casos buenas, 
no sucede lo mismo con la protección al postulante explorado, ya que recibe una "dosis" directa que, mientras la reciba una vez al año, no sería tóxica $\left({ }^{14}\right)$. Sin embargo, desde que aparece la legislación para la liberación del trabajo por services, el postulante es sometido varias veces al año a exploración torácica con rayos equis mediante telerradiografía y, lo que es peor, algunas veces con fotorradiografía. Lamentablemente, la utilidad de estas exploraciones son cuestionadas, sobre todo cuando se las hace en forma rutinaria $\left({ }^{16}\right)$. De ellas, Felson citado por Vielba $\left({ }^{17}\right)$ dice: "Estoy cansado de que me hagan interconsultas mostrándome en los negatoscopios solamente radiografías AP de tórax. No hay oblicuas ni laterales ni penetradas con Bucky, ni localizadas... en esta situación miro las radiografías desesperado pensando: ¿Cómo puedo hacer un diagnóstico con tan poca información?".

En minería, este estudio es indispensable y una obligación legal $\left({ }^{25}\right)$. Pero, aún así, el uso indiscriminado de cualquier sistema de exploración con rayos $\mathrm{X}$ es potencialmente perjudicial para el trabajador $\left({ }^{18}\right)$.

En el Perú todavía tenemos empresas de la gran minería que usan foto radiografía de 70 ó $100 \mathrm{~mm}$ para el examen periódico de sus trabajadores expuestos a polvo, sin considerar el mayor riesgo para el trabajador examinado, para el operador del equipo y la poca resolución de la placa, aún leyéndola con lupa de gran aumento. Pero, su costo definitivamente es menor, sobretodo cuando se toma 2 ó 3 mil placas. Con el mismo argumento utilizan este método para exámenes pre empleo $\left({ }^{24}\right)$. Y cuando nuestros trabajadores enferman o mueren de cáncer, a nadie se le ocurre preguntar "por qué de cáncer". Es más con nuestro sistema de información en Salud, es imposible hacer el seguimiento retrospectivo para determinar exposiciones ocupacionales a tóxicos que expliquen el deceso.

El Sievert (Sv) es un término especial adoptado en 1979 por la XVI Conferencia General de Pesas y Medidas, para designar la unidad
SI de dosis equivalente usada en higiene industrial para la protección contra las radiaciones, aunque médicamente seguimos adhiriendo a lo que sostiene la OMS sobre la inexistencia de valor límite umbral $\left({ }^{31}\right)$. Un Sievert es igual a 100 Rem.

La dosis colectiva de una población se expresa en Sievert-persona o Rem-persona y representa el promedio de dosis persona por el número de personas expuestas; así, un Sievert actuando sobre 100 personas equivale a 100 Sievert-persona o a 10000 Rem-persona.

Band y colaboradores establecen que aproximadamente el $13 \%$ de la radiación ionizante total recibida por el hombre a nivel del mar proviene de la radiación cósmica. Esta cantidad se incrementa con cada $1800 \mathrm{msnm}$ adicionales, debido a la menor densidad de la capa atmosférica protectora $\left({ }^{23}\right)$. Se ha calculado que la dosis promedio anual de este tipo de radiación recibida por la población norteamericana es de 31 $\left.\mathrm{mRem} / \mathrm{año}{ }^{(32}\right)$.

Se ha demostrado que los efectos de exposición a dosis bajas son tardíos y además que el exceso total de todo tipo de cáncer por mil personas por Sv / año, comienza entre 5 a 10 años después de la exposición y continúa durante toda la vida. El efecto acumulativo en tiempo de vida total se ha estimado en un exceso de aproximadamente 20 a 100 casos de cáncer por 1000 expuestos por $\mathrm{Sv}$, lo que corresponde a un incremento en el riesgo de 10 al $60 \%$ por Sv para el promedio del tiempo de vida actual $\left({ }^{15}\right)$.

Tomando en cuenta que para una radiografía estándar de pulmones el riesgo calculado es de 1,6 casos por millón de exámenes, si lo multiplicamos por el número de radiografías tomadas en el año, el riesgo se multiplica por este mismo número.

Nuestra investigación demuestra que durante el lapso estudiado el trabajador que ingresó a laborar en services fue expuesto por este motivo hasta en 7 oportunidades a radiación equis y recibió en promedio $48 \mathrm{mRem} / \mathrm{año}$ (valores 
extremos 12 y 84). Además, por la naturaleza misma del trabajo, se expuso a una dosis adicional de radiación natural de 77,5 mRem/año. Si agregamos la radiación que recibió para el diagnóstico de enfermedades y accidentes de trabajo, una media de $430 \mathrm{mRem} / \mathrm{año}$, tenemos un promedio pesado de dosis equivalente efectiva año de $169 \mathrm{mRem} / \mathrm{año}$, lo que incrementó el riesgo total promedio para contraer cáncer a $289 \times 10^{-6}$ exámenes. El riesgo es mayor en el grupo de trabajadores, a quienes además de las radiografías de tórax de ingreso se les realizó exámenes radiográficos de parrilla costal; aquí el riesgo se incrementó a 793 x 10-6 exámenes. Contrariamente, el menor riesgo fue para el grupo que sólo tuvo exámenes radiográficos adicionales de abdomen simple, 26,8.

Indudablemente, desde el punto de vista de Salud Ocupacional, no podemos controlar la radiación ionizante natural, por lo que nuestras acciones se deben orientar a controlar los riesgos de exposición que dependen del uso médico de rayos equis. En el aspecto de la frecuencia de accidentes, sí es posible coordinar con traumatólogos e internistas para que limiten su uso o empleen otros métodos diagnósticos y no la radiación.

Donde sí podemos intervenir como médicos ocupacionistas es en la reducción del riesgo por exámenes de ingreso al trabajo, pues escapa a todo criterio médico, lógico o técnico que se tome 6 ó 7 radiografías de pulmones en un año a supuestos sanos que postulan a trabajo. Es necesaria la pronta reglamentación de estas leyes, ya que actualmente son aplicadas sin criterio. Un examen, y una radiografía de tórax, debería ser válido por lo menos durante un año, tal como lo es para los exámenes periódicos de trabajadores de planta, pues esta periodicidad es válida también para aquellos ya diagnosticados de neumoconiosis.

Por el análisis de los resultados concluimos que, en la población estudiada, a $45 \%$ de los examinados se les toma una radiografía de tórax al año, a $29 \%$ dos, a $12 \%$ tres, a $13 \%$ entre cuatro y seis y a 5 postulantes se les toma 7 radiografías en el año. La dosis equivalente efectiva promedio por este motivo es $48 \mathrm{mRem} / \mathrm{año}$. Por otros exámenes radiográficos, la población recibe una media de $430 \mathrm{mRem} /$ año y por radiación natural un promedio de 77,5 mRem/año. El promedio pesado anual de dosis equivalente efectiva acumulada de radiación ionizante que encontramos es del orden de los $169 \mathrm{mRem} / \mathrm{año}$ y el promedio pesado de riesgo total de contraer cáncer es de $289 \times 10^{-6}$ exámenes.

Por último, y porque en la población estudiada hemos encontrado sobre exposición a radiación ionizante, debido principalmente a la gran cantidad de exámenes radiográficos a que se le somete, recomendamos se racionalice el número de exámenes de ingreso al trabajo $\mathrm{y}$, por tanto, el número de exámenes radiográficos.

\section{BIBLIOGRAFÍA}

1. World Health Organization. Effect of ionizing and nonionizing radiation. Report of a Working Group. Copenhagen. 1972.

2. Organización Mundial de la Salud. Comité de Expertos en Tuberculosis, $9^{\circ}$ Informe. Ginebra 1974.

3. International Commission on Radiological Protection. Non stochastics effects of ionizing radiation. ICRP Publication 41, Annals of the IRCP, 1 984; 14(3).

4. Neel JV. Update on the genetic effects of ionizing radiation. J Am Med Ass 1991; 266: 698.

5. United Nations Scientific Committee on Effects of the Atomic Radiation. Ionizing radiation: sources and biological effects. Report to the General Assembly. Annexes. United Nations NY 1982.

6. International Commission on Atomic Energy Agency. What the general practitioner (MD) should know about medical handling of exposed individuals. IAEA - Technical Document No 306. Genev 1986.

7. International Commission on Radiological Protection (ICRP). Recommendations of the International Commission on Radiological Protection. ICRP Publication 26. Annals of the ICRP 1977; 1(3).

8. Kitabatake, MD. Estimation of benefit and radiation risk from mass chest radiographic. Radiol East 1973; 109: 37-40.

9. Greeg, EC. Risk/benefit considerations on chest radiography. En: Physics of Diagnostic Radiology. Proceedings of a Summer School. Publication FDA - 74 8007. Washington 1974. 
10. Nogueira DP. Abreugrafia e o risco das radiaçôes ionizantes. Rev Bras Saúde Ocupacional 1985; 50(13): 7-13.

11. Peixoto JE. Risco de neoplasias associado ao exame de abreugrafia. Rev Bras Saúde Ocupacional 1985; 50(13): 14-8.

12. Hashizume T, Maruyama R. Estimation of the stochastic risk from medical X-ray diagnosis. Nippon Acta Radiol 1979; 39: 170-3.

13. Jankowsky J. Evaluation of the risk of the neoplasm induction in the Polish populations in result of X-ray radiation applied for medical purposes. Lodz 3. Polish, 1982.

14. International Commission on Radiological Protection (ICRP). Recommendations of the International Commission on Radiological Protection. ICRP Publication 60. Pergamon Press 1991.

15. Levy VS, Wegman DH. Occupational Health Recognizing and Preventing Work-Related Disease. Boston, USA: Little, Brown and Co 1988.

16. Vielba JA. Problemática actual de la fotoseriación en los reconocimientos médicos laborales. Med Segur Trabajo, 1988; XXXV (140): 65-9.

17. National Center for Devices and Radiological Health. NCDRH/ FDA Panel Report, USA. 1983.

18. Committee on the Biological Effect of Ionizing Radiation. Health effects of exposure to low levels of ionizing radiation BEIR V. National Research Council. National Academy Press 1990.

19. United Nations Scientific Committee on Effects of the Atomic Radiation. Ionizing radiation: sources and biological effects. Report to the General Assembly , NY United Nations 1977.

20. Rall JE, Beebe GW, Hoel DE, et al. Report of the National Institutes of Health Working Group to Development Radio
Epidemiological Tables. NIH Publication No 85: 2748. US Government Printing Office. Washington DC. 1985.

21. Balter M. Chernobyl's thyroid cancer toll. Science 1995; 270: 1758

22. La Dou J, Jackson RT, Howard JJ. Exposiciones y controles ambientales. En: J La Dou. Medicina Laboral y Ambiental, 2 ${ }^{a}$ Edición. México: Ed. Manual Moderno 1999.

23. Band PR, et al. Mortality and cancer incidence on a cohort of commercial airline pilots. Space Environ Med 1990; 61: 299.

24. Ramírez AV. Condiciones de salud en el trabajo minero metalúrgico en Perú. Memorias del XIX Congreso de Medicina del Trabajo y Salud Ocupacional. ALSO, Cartagena de Indias, Colombia. Setiembre 1999.

25. Ministerio de Energía y Minas del Perú. Reglamento de Seguridad e Higiene Minera, DS 03-94 EM. Lima. Perú. 1994.

26. Pastore J. A agonia do emprego, $1^{\text {a }}$ Ed. Sao Paulo Brasil: Editorial LTR 1997.

27. Knights D, Willmot H, Collinson D. Job Redesign. Gover, Aldershot. 1990.

28. Ministerio de Trabajo del Perú. Ley de Productividad y Competitividad Laboral, DS 003-97- TR. Lima. Perú. 1997.

29. Bezerra AM. Os novos paradigmas de Organizaçâo do Trabalho: Implicaçôes na Saúde Mental dos trabalhadores. Rev Bras Saúde Ocupacional 1997; 85-86 (23): 55-60.

30. Repullo EJ. Os sindicatos a terceirizaçâo e a saúde dos trabalhadores. Rev Bras Saúde Ocupacional 1997; 85-86 (23): 79-82.

31. Organización Mundial de la Salud. Las Unidades SI para las profesiones de salud. OMS Ginebra, Suiza. 1980.

32. Holland HD, Petersen V. Living Dangerous. Princenton University Press. 1995. 